\title{
Escala de Aconselhamento Profissional: ANÁlise COM ESTUdANTES DE Ensino Médio
}

\author{
Ana Paula Porto Noronha \\ Maiana Farias Oliveira Nunes $\star \star$
}

\begin{abstract}
Resumo
Este estudo teve como objetivo analisar a Escala de Aconselhamento ProfissionalEAP com alunos do ensino médio, buscando fazer comparações com a amostra normativa, composta por estudantes universitários. Dois conjuntos de dados foram utilizados, um deles composto por 762 estudantes universitários, e o segundo com 950 alunos do ensino médio. Análises fatoriais e de Rasch foram realizadas, tendose observado a mesma estrutura fatorial que havia sido previamente definida para estudantes universitários com a nova amostra, composta por adolescentes. A precisão dos itens variou de 0,97 a 0,99, enquanto a precisão das pessoas variou entre 0,70 e 0,85. Os parâmetros de ajuste estiveram em patamares adequados tanto em relação às pessoas como com os itens. O theta e a dificuldade dos itens foram semelhantes em ambas as amostras. Os resultados foram interpretados como favoráveis ao uso de EAP para medir os interesses vocacionais dos adolescentes, considerando as dimensões previamente definidas.
\end{abstract}

Palavras-chave: interesses profissionais; adolescentes; teste psicológico

\section{Professional Counseling Scale: ANALYSIS WITH HigH SCHOOL STUDENTS}

\begin{abstract}
This study aimed at analyzing the "Escala de Aconselhamento ProfissionalEAP" with high school students, trying to make comparisons with the normative sample that answered the scale, which was composed by college students. Two data sets were used, the first one composed by 762 college students, and the second one comprised of 950 high school students. Factor and Rasch analyses were conducted. The same factor structure defined for college students was found with adolescents successfully. Item reliability ranged from 0.97 to 0.99 and people reliability varied between 0.70 and 0.85 ; Fit statistics, regarding people

^Psicóloga. Doutora em Psicologia Ciência e Profissão pela Pontifícia Universidade Católica de Campinas. É professora associada doutora da Universidade São Francisco. Endereço: Universidade São Francisco. Av. Senador Lacerda Franco, Sn - Itatiba, SP - Brasil. CEP:13251-900.

E-mail: ana.noronha@saofrancisco.edu.br

$\star \star$ Psicóloga. Realizou pós doutorado em Psicologia na Universidade Federal do Rio Grande do Sul, no departamento de Psicologia do Desenvolvimento e da Personalidade, com bolsa do Cnpq de PDJ.

E-mail: maiananunes@mac.com
\end{abstract}


and items, was adequate. Theta and item difficulty were similar in both samples. Results were interpreted as favorable to using EAP to measure vocational interests of adolescents, considering the previously defined dimensions.

Keywords: vocational interests; adolescents; psychological test

\section{INTRODUÇão}

A Teoria de Resposta ao Item (TRI) vem sendo referenciada como uma das correntes para análise de testes psicológicos. Ela fundamenta-se no pressuposto de que, ao se conhecer as características observadas (respostas aos itens de um teste), essas entram em equações matemáticas como constantes e, assim, torna-se possível conhecer ou estimar o nível de habilidade do sujeito. O inverso dessa afirmação é verdadeiro, ou seja, ao conhecer o nível de habilidade do sujeito, é possível estimar a dificuldade do item. Dentro dessa abordagem, o traço latente (variável psicológica inferida, não observável diretamente) é chamado de theta (EMBRETSON; REISE, 2000, PASQUALI, 2003; PASQUALI; PRIMI, 2003, WRIGHT; STONE, 2004).

Embretson e Reise (2000) apresentam a TRI como um modelo de medida em que o nível das estimativas dos thetas depende tanto das respostas das pessoas, como das propriedades dos itens utilizados. Nesse modelo, pessoas com o mesmo theta são agrupadas e, a partir desses agrupamentos, busca-se modelar o seu padrão de resposta a um conjunto de itens. Assume-se que cada examinando possui um dado nível de habilidade, que permite situá-lo em uma posição específica em uma escala de habilidade. Vale notar que para cada nível de habilidade existe uma probabilidade específica de acerto ou de adesão ao conteúdo expresso no item.

São considerados, na TRI, modelos de um, dois ou três parâmetros para a análise de itens (parâmetros a, b e c), que são, respectivamente, discriminação, dificuldade e probabilidade de acerto aos itens ao acaso. O parâmetro de discriminação diz respeito à capacidade de um item diferenciar pessoas com níveis diferentes de habilidade, informando sobre a sensibilidade da escala em determinadas áreas da curva de informação dos itens. Esse parâmetro indica o quanto um item pode diferenciar pessoas abaixo da localização da dificuldade do item e acima dela. Já a dificuldade do item informa a região de theta na qual o item funciona melhor ao longo da escala de habilidade, sendo assim um índice de localização dos itens, ou seja, indica para quais níveis de habilidade o item funciona mais adequadamente. Em regiões de theta com pessoas com menor habilidade, há uma menor probabilidade de acerto a itens difíceis, ou seja, seria necessário um maior nível de habilidade para acertar o item. À medida que o theta aumenta, amplia-se a probabilidade de acerto ou adesão ao conteúdo semântico do item. Já itens com menor nível de dificuldade podem ser acertados tanto por pessoas com thetas maiores, como por pessoas com thetas menores. Por fim, o parâmetro c, que aborda a chance de acerto ao acaso, informa sobre a probabilidade de um sujeito acertar o item, mesmo sem o nível necessário de habilidade para tal. Esse 
parâmetro só faz sentido em testes que são corrigidos em termos de acerto e erro, e não em escalas de autorrelato em que as pessoas indicam o grau de concordância com frases, por exemplo (BAKER, 2001).

Para o presente artigo, foi utilizado o modelo de um parâmetro ou o modelo de Rasch, que considera a dificuldade dos itens (RASCH, 1960). Considerando esse modelo, alguns índices foram analisados nesse artigo, quais sejam, ajuste dos itens (infit e outfit), dificuldades dos itens, theta, precisão das dimensões e o erro padrão de theta e da dificuldade dos itens. O ajuste dos itens refere-se à consistência de respostas dos sujeitos a diferentes itens, de acordo com o seus níveis de habilidade. Para calcular esse índice, compara-se o total de respostas dos sujeitos para um item específico, a fim de verificar se há uma coerência (consistência) no padrão de respostas para pessoas com níveis semelhantes de habilidade. Por exemplo, ocorre desajuste quando um item muito difícil é acertado por pessoas com baixa habilidade. Dois índices de ajuste foram estudados nessa pesquisa, o outfit e o infit. O primeiro informa sobre a ocorrência de respostas inesperadas nos casos extremos (outliers). Nesse caso, é verificada discrepância entre o padrão de respostas esperado e observado num nível muito distante da habilidade do sujeito, ou seja, ele erra itens muito fáceis para sua habilidade ou acerta itens muito difíceis para sua habilidade. Já o infit é menos sensível aos outliers e mais sensível a problemas estruturais nos itens. O infit revela se há um desajuste no padrão de respostas em itens próximos do nível de habilidade dos sujeitos, ou seja, pode ocorrer quando pessoas com um nível de habilidade semelhante à dificuldade do item sistematicamente erram o item (BAKER, 2001).

O erro padrão, por sua vez, é a variabilidade aleatória, não prevista pelo modelo de Rasch, analisado tanto no que se refere ao theta das pessoas como à dificuldade dos itens. A precisão é outro indicador da consistência das respostas, que permite avaliar a quantidade de erro contida nas medidas. No modelo de Rasch é avaliada a precisão das pessoas e dos itens, e também a precisão "real" e "modelada", sendo esses os valores de limite inferior e superior das precisões, respectivamente. Assim, o "escore verdadeiro" dos sujeitos, ou seja, seu escore livre de erro de medida, deve se encontrar em algum lugar entre o limite inferior e superior da precisão real e modelada (LINACRE, 1997).

Apesar de a TRI ser mais usualmente empregada em testes de habilidade, alguns pesquisadores utilizaram o modelo de Rasch com construtos que são avaliados com itens em escalas intervalares e objetivaram, por exemplo, a avaliação da personalidade (NUNES et al., 2008a) ou de sintomas psiquiátricos variados, como depressão e transtorno paranoide (ELLIOT et al., 2006), o que justifica o uso no presente estudo. A presente pesquisa voltou-se para a análise do construto interesses profissionais, que será discutido em sequência. Para compreender a análise de Rasch aplicada a testes de interesse, o theta não deve ser compreendido como "habilidade", mas sim como o traço "interesses profissionais", do mesmo modo que a "dificuldade dos itens" deve ser entendida como a adesão dos sujeitos ao conteúdo semântico expresso nos itens. 
Quanto ao conceito de interesse, não há na literatura, e não se esperava que fosse diferente, um consenso quanto à melhor definição do construto. Ao contrário, encontra-se nesse campo de investigação, assim como nas demais áreas da psicologia, muitas concepções distintas e algumas antagônicas. Para Mattiazzi (1977), não há exatamente uma teoria sobre os interesses; há tentativas de explicação do fenômeno, que são confirmadas ou não por meio das pesquisas científicas. $\mathrm{O}$ autor acrescenta que os interesses são fenômenos psicossociais, complexos e decorrentes de vários fatores, o que dificulta a compreensão deles.

A confusão atribuída à definição do construto foi reconhecida por diversos autores, como Bohoslavsky (1993), Leitão e Miguel (2004), Nunes et al. (2008b), dentre outros. Mattiazzi (1977), há quase 30 anos, chamava a atenção para o fato de que embora a literatura estrangeira sobre o tema fosse extensa, no Brasil encontrava-se de maneira incipiente.

De acordo com o autor, o despertar do estudo sobre o interesse se deu, mais especialmente, no início do século XX, quando sociólogos e economistas estavam preocupados com o aumento da produtividade e da economia. Em razão da ausência de conjuntos teóricos sistematizados, as pesquisas empíricas ganharam espaço, o que se mantém nos dias atuais, tal como destacado por Noronha e Ambiel (2006), por Noronha et al. (2006) e por Teixeira et al. (2007).

Um dos marcos do estudo sobre interesses se deu com Fryer (1931), com a publicação da obra The measurement of interests, cuja relevância se mantém. Para ele, há os interesses subjetivos e os objetivos, sendo que os primeiros são compreendidos como gostos e experiências acompanhados por sentimentos de satisfação; ou não gostos e afirmações de aversões acompanhadas de sentimentos desagradáveis. Já os interesses objetivos são entendidos como reações, comportamentos que podem ser observados por outros em uma situação específica, sendo que ambos (objetivos e subjetivos) são atividades de aceitação-rejeição e prazerdesprazer. De acordo com o autor, a aprendizagem favorece o desenvolvimento dos interesses. Apoiando-se no modelo de S-R (estímulo-resposta), o autor defendia que o indivíduo aprende a aceitar ou rejeitar certos objetos e atividades, de acordo com a experiência que tem com eles.

Strong (1943) compartilha da ideia de que os interesses podem ser aprendidos e modificados pela reeducação. Se um indivíduo tem a habilidade e a vontade de adquirir um interesse, pode vir a alcançar o que deseja. Assim, aceita-se a ideia de que é possível favorecer o surgimento de um interesse, uma vez que ele se desenvolve quando alguém encontra satisfação de seus desejos. Em síntese, as atividades executadas com sucesso para satisfazer alguma necessidade tendem a se tornarem interessantes.

Os interesses são entendidos como um dos fenômenos psicológicos que favorecem o ajustamento do indivíduo às condições ambientais, segundo Carter (1940). Sob esta perspectiva, os interesses auxiliariam as pessoas nas soluções de problemas de ajustamento, por meio de estratégias de adaptação, o que é endossado posteriormente por Angelini (1957). Assim, entende-se que a compreensão 
dos interesses é socialmente relevante, uma vez que permite ao psicólogo a análise de uma característica que pode permitir a busca de bem-estar por parte de seus clientes, considerando o contexto do trabalho.

Mais contemporaneamente, Savickas (1999) definiu o construto como um estado de "consciência", caracterizado por prontidão de respostas a estímulos ambientais específicos. Em um trabalho anterior, Savickas (1995), ao definir o conceito, faz referência ao padrão de gostos, desgostos e respostas indiferentes a estímulos do ambiente. Convém destacar que Leitão e Miguel (2004) referem-se à definição de Savickas (1999) como a que melhor integra as ideias concernentes ao construto.

De acordo com o último autor, as teorias de interesse podem ser agrupadas em três conjuntos. No primeiro são reunidas as concepções embasadas na teoria da aprendizagem, de tal modo que os indivíduos aprendem a gostar das atividades que fazem bem. Lent, Brown e Hackett (1994) são representantes deste grupo, defendendo a ideia que crianças aprendem padrões de aceitação ou rejeição de estímulos ocupacionais pela experimentação, pela observação do outro ou por meio dos feedbacks.

No segundo grupo, ainda segundo Savickas (1999), estão incluídas as abordagens que envolvem personalidade e motivação. Sob essa perspectiva, os interesses estariam a serviço do desenvolvimento da personalidade. Dentre os teóricos que mais se destacaram convém mencionar Carter (1940), que defendia que os interesses promovem a integração da personalidade, tal como já assinalado, ou Bordin (1943), que considerava o interesse como a expressão do autoconceito ocupacional.

Por fim, a terceira categoria enfatiza o papel social associado aos interesses, sendo que a aceitação ou rejeição do papel social gera o gosto ou o desgosto de atividades. Como referência dessa abordagem, cita-se Tyler (1951). No tocante à última concepção, a imagem de uma ponte que une o papel social ao papel individual tem sido uma metáfora útil. Savickas (1995) destaca que essa ideia coincide com a etimologia do termo; mais especialmente, interesse, no latim, significa "entre ser/estar". Assim, o construto representaria a ligação entre o indivíduo e o mundo. Sob essa perspectiva, os interesses ajudariam na manutenção da integridade do indivíduo, por favorecer a prática de ajustamento às condições ambientais, em um processo de máximo de "auto-completamento".

Os interesses são compreendidos por Holland $(1959,1963)$ como resultantes da associação com a personalidade do indivíduo. Em acréscimo, para o autor, a congruência entre personalidade e ambiente produz bons resultados, como satisfação e realização no trabalho. Posteriormente, Holland, Powell e Fritzsche (1994) propuseram que as pessoas aprendem a gostar mais de certas atividades que outras, o que pode ser explicado pelas tendências motivacionais. Assim, segundo os autores, as pessoas tendem a obter maior satisfação no trabalho quando conseguem estar em ambientes profissionais com as mesmas características que definem seu interesse. 
Um dos instrumentos que avaliam interesses profissionais, disponível para uso profissional no Brasil para a população universitária, é a Escala de Aconselhamento Profissional (EAP) (NORONHA; SISTO; SANTOS, 2007). Os autores da EAP compreendem interesses como padrões de preferências por atividades profissionais específicas, coerentemente com as proposições de Savickas (1995), que, por sua vez, sugere que o construto refere-se ao padrão de gostos, desgostos e respostas indiferentes quanto a estímulos ocupacionais.

Os objetivos desse estudo envolveram a análise psicométrica do EAP em alunos de ensino médio, buscando verificar se a interpretação sugerida no manual, indicada para universitários, também se mantém pertinente quando se considera uma população mais jovem. Mais especificamente, realizou-se a análise fatorial do teste e análises de Rasch com esse novo grupo. Foi verificado, por meio da comparação com a estrutura fatorial original, se ela se manteve e se os mesmos itens comporiam os mesmos fatores. As análises de Rasch incluíram a verificação do ajuste dos itens das dimensões (infit e outfit), dificuldade dos itens e theta das pessoas, erro padrão das estimativas dos itens e das pessoas, além da precisão das dimensões. Adicionalmente, vale destacar que as análises de Rasch foram realizadas comparando-as com aquela realizada com universitários. No que diz respeito à análise de Rasch realizada com o EAP respondida por universitários, descrita no manual do teste, os autores da escala afirmam que, de forma geral, os índices de ajuste das dimensões encontraram-se adequados (NORONHA; SISTO; SANTOS, 2007; SISTO; NORONHA; SANTOS, 2011).

No que diz respeito à comparação dos dados da EAP com universitários e alunos do ensino médio, uma discussão pertinente envolve a questão de se há diferenças nos padrões de interesse associadas à idade ou à série escolar. Nesse específico, um estudo realizado com a EAP aplicada em estudantes do ensino médio, indicou que apenas uma dimensão da escala (denominada Artes e Comunicação) teve diferença de média significativa associada à série escolar (SARTORI; NORONHA; NUNES, 2009). No entanto, as autoras destacaram que essa diferença deveria ser mais investigada, uma vez que a amostra constitui-se por jovens apenas do estado de São Paulo, sendo todos alunos de escolas particulares. Adicionalmente, os autores de destaque na análise dos interesses indicam não existir diferenças na configuração dos interesses associadas à idade (exemplo: HOLLAND, 1959; LENT; BROWN; HACKETT, 1994; SAVICKAS, 1995, 1999). Desse modo, presentemente esperava-se que a estrutura fatorial dos interesses encontrada na EAP em universitários se manteria, quando analisadas as respostas de jovens de ensino médio.

\section{MÉTodo}

\section{Fonte de dados}

Para a realização da presente pesquisa contou-se com duas fontes para a análise, um banco de dados com as respostas dos universitários, cedido pelos autores do EAP, e outro banco, advindo da aplicação em uma amostra de ensino médio. Ambos os bancos foram cedidos pelos autores e os dados dos participantes serão descritos separadamente a seguir. 
Os dados normativos do EAP foram obtidos a partir da aplicação em 762 universitários, de 12 cursos distintos. Entre eles, a maior participação foi de alunos de psicologia $(21,9 \%)$, seguida por engenharia $(11,4 \%)$ e administração $(10,4 \%)$. As idades variaram entre 17 e 73 anos, com média de 24,1 e desviopadrão de 7,1. A maioria dos participantes era mulher (59\%), sendo que a coleta foi principalmente realizada no estado de São Paulo.

A outra base de dados foi composta por 950 alunos do ensino médio de escolas públicas e particulares dos estados de São Paulo ( $\mathrm{N}=836)$ e do Paraná $(\mathrm{N}=114)$. Os jovens possuíam entre 14 e 20 anos, com média de idade de 16,3 anos e desviopadrão de 0,9 . Quanto às séries que cursavam, quatro participantes não indicaram essa informação, $23 \%$ eram da primeira série, $30,3 \%$ da segunda e $46,7 \%$ da terceira. No que diz respeito ao sexo, $64,7 \%$ eram mulheres e $35,3 \%$, homens.

\section{Instrumento}

Foi utilizada a Escala de Aconselhamento Profissional, que avalia preferências profissionais. A EAP foi construída empiricamente com base em guias de profissões e descrições de perfis profissionais oferecidos na internet por universidades brasileiras. Para a seleção inicial de itens foi realizada a análise heurística. Esses procedimentos permitiram a seleção de 61 itens que descrevem atividades, que são avaliadas em termos da frequência que o sujeito gostaria de desenvolvêlas em escala likert de 1 a 5.

A análise fatorial da EAP, descrita no manual, indicou a existência de sete fatores, com autovalores superiores a 1,5, que juntos, explicaram $57,3 \%$ da variância. Todos os itens apresentaram saturação superior a 0,3 e nenhum deles precisou ser eliminado. Os fatores foram denominados Ciências Exatas, Artes/ Comunicação, Ciências Biológicas e da Saúde, Ciências agrárias/ambientais, Burocráticas, Ciências Humanas/Sociais aplicadas e Entretenimento. Os fatores apresentaram quantidades desiguais de itens e, em alguns casos, o mesmo item participou de mais de um fator (NORONHA; SISTO; SANTOS, 2007).

\section{Procedimento de coleta de dados}

Uma vez que o presente estudo contou com duas bases de dados, não é possível detalhar a maneira que os dados foram coletados. Contudo, uma informação disponível é que, em ambas amostras, os dados foram coletados coletivamente, em sala de aula, por psicólogos ou alunos de psicologia treinados para a aplicação da EAP. Todos os participantes maiores de idade assinaram o Termo de Consentimento Livre e Esclarecido e, no caso de menores de idade, seus responsáveis legais o fizeram.

\section{Resultados}

Inicialmente foi verificada a possibilidade de fatoração da EAP por meio da análise do KMO e do teste de Esfericidade de Bartlett com a amostra de ensino médio. O KMO observado foi de 0,90 e o teste de Bartlett foi significativo ( $p<$ 
0,001), indicando a possibilidade de extração dos fatores. Com caráter exploratório, inicialmente realizou-se a análise fatorial sem determinar a quantidade de fatores desejada, verificando-se que doze fatores apresentavam autovalor superior a 1 . No entanto, como os objetivos desse artigo envolveram verificar se a estrutura original da escala se manteria, as análises subsequentes consideraram a determinação prévia de extração de sete fatores. Os sete fatores explicaram conjuntamente $54,1 \%$ da variância e apresentaram autovalor superior a 1,4. Em seguida, foi feita a análise dos componentes principais solicitando a extração de sete fatores, com rotação Promax, com itens com carga fatorial superior a 0,30, seguindo os mesmos procedimentos descritos no manual do EAP. A análise da matriz da estrutura é apresentada na Tabela 1.

Tabela 1- Matriz de estrutura da EAP com alunos do Ensino Médio

\begin{tabular}{|c|c|c|c|c|c|c|c|}
\hline Item & $\mathrm{AC}$ & $\mathrm{CE}$ & CAA & CBS & $\mathrm{AB}$ & $E$ & CHSA \\
\hline 45 & 0,82 & & & & & 0,31 & \\
\hline 44 & $\mathbf{0 , 8 0}$ & & & & & 0,36 & \\
\hline 35 & 0,77 & 0,30 & & & & 0,35 & \\
\hline 46 & 0,75 & & & & & 0,38 & \\
\hline 24 & 0,67 & & & & & 0,47 & \\
\hline 43 & 0,67 & & & & & 0,32 & \\
\hline 56 & 0,63 & 0,43 & & & & 0,35 & \\
\hline 29 & 0,60 & 0,33 & & & & & \\
\hline 20 & 0,58 & & & & & & $\mathbf{0 , 5 0}$ \\
\hline 26 & 0,56 & & & & & & $\mathbf{0 , 5 3}$ \\
\hline 27 & 0,54 & & & & 0,31 & & 0,35 \\
\hline 28 & 0,53 & 0,36 & & & & 0,36 & \\
\hline 53 & 0,52 & 0,37 & & & & & \\
\hline 33 & 0,47 & & & & 0,44 & & \\
\hline 60 & & 0,82 & & & & & \\
\hline 10 & & 0,81 & & & & & \\
\hline 14 & & 0,79 & & & & & \\
\hline 31 & & 0,76 & 0,38 & & & & \\
\hline 34 & & 0,68 & & & & & \\
\hline 13 & & 0,65 & & & & & \\
\hline 59 & & 0,63 & & & 0,33 & & \\
\hline 7 & & 0,62 & 0,31 & & 0,32 & & \\
\hline 55 & & $\mathbf{0 , 5 8}$ & & & 0,52 & & \\
\hline 32 & & $\mathbf{0 , 5 3}$ & 0,47 & & & & \\
\hline 41 & & & 0,78 & & & & \\
\hline 17 & & & 0,76 & 0,32 & & & \\
\hline
\end{tabular}




\begin{tabular}{|c|c|c|c|c|c|c|c|}
\hline Item & $\mathrm{AC}$ & $\mathrm{CE}$ & CAA & CBS & $\mathrm{AB}$ & $\mathrm{E}$ & CHSA \\
\hline 50 & & & 0,75 & & & & \\
\hline 12 & & 0,35 & 0,72 & & & & \\
\hline 51 & & & 0,70 & & & & \\
\hline 25 & & & 0,68 & & & & \\
\hline 40 & & & 0,64 & 0,63 & & & 0,33 \\
\hline 11 & & 0,36 & 0,63 & & & & \\
\hline 18 & & & 0,63 & 0,44 & & & \\
\hline 37 & & & 0,56 & & & 0,42 & \\
\hline 19 & & & 0,45 & & 0,36 & & \\
\hline 36 & & & 0,43 & 0,37 & & & \\
\hline 3 & & & & $\mathbf{0 , 8 3}$ & & & \\
\hline 6 & & & & $\mathbf{0 , 8 2}$ & & & \\
\hline 42 & & & & 0,76 & & & \\
\hline 8 & & & & 0,74 & & & \\
\hline 39 & & & & 0,71 & & & \\
\hline 38 & & & $\mathbf{0 , 5 1}$ & 0,65 & & & 0,31 \\
\hline 49 & & & 0,44 & 0,64 & & & \\
\hline 58 & & & 0,59 & 0,62 & & & \\
\hline 48 & & & 0,39 & 0,40 & & & \\
\hline 47 & & & & & & & \\
\hline 15 & & & & & 0,74 & & \\
\hline 30 & & & & & 0,72 & & \\
\hline 54 & & & & & 0,72 & & \\
\hline 1 & & & & & 0,71 & & \\
\hline 23 & & & & & 0,69 & & \\
\hline
\end{tabular}




\begin{tabular}{|c|c|c|c|c|c|c|c|}
\hline Item & $\mathrm{AC}$ & $\mathrm{CE}$ & CAA & CBS & $\mathrm{AB}$ & $\mathrm{E}$ & CHSA \\
\hline 21 & & $\mathbf{0 , 4 9}$ & & & 0,60 & & \\
\hline 22 & & $\mathbf{0 , 3 2}$ & & & $\mathbf{0 , 5 0}$ & & \\
\hline 9 & 0,45 & & & & & 0,79 & \\
\hline 4 & 0,47 & & & & & 0,75 & \\
\hline 61 & 0,45 & & & & & 0,74 & \\
\hline 2 & 0,45 & & & & & $\mathbf{0 , 7 0}$ & \\
\hline 52 & & 0,43 & & & $\mathbf{0 , 3 7}$ & 0,48 & \\
\hline 16 & & & & & & & $\mathbf{0 , 8 0}$ \\
\hline 5 & & & 0,35 & & & & 0,75 \\
\hline 57 & & & & & 0,31 & & 0,65 \\
\hline
\end{tabular}

Legenda: AC (Artes e Comunicação), CE (Ciências Exatas), CAA (Ciências agrárias e ambientais), CBB (Ciências Biológicas e da Saúde), AB (Atividades Burocráticas), E (Entretenimento), CHSA (Ciências Humanas e Sociais aplicadas). As cargas fatoriais em negrito indicam os casos em que há correspondência com a estrutura da escala original.

Por meio da comparação entre a matriz de estrutura da EAP com pessoas do Ensino Médio (EM) e Universitário, verificou-se a manutenção dos fatores, com os mesmos itens que os compuseram originalmente, embora tenham sido encontradas algumas poucas diferenças. Foi possível observar que determinados itens possuíam carga fatorial superior a $0,30 \mathrm{em}$ mais de um fator, e, do mesmo modo que foi realizado no manual do EAP, um mesmo item pôde ser mantido em mais de um fator, considerando que a mesma atividade profissional pode estar presente em mais de uma área. Em alguns casos (exemplos: itens 52 e 56), optouse por manter os itens nos fatores originais e não em novos fatores, ainda que a carga fatorial do item fosse mais elevada em um fator diferente do proposto pelos autores do teste. Como o objetivo dessa análise foi verificar se a estrutura original poderia ser mantida com uma amostra com características distintas da original, considerou-se o resultado satisfatório, no que se refere à manutenção dos mesmos itens das dimensões originais.

Tendo feito essa análise da estrutura fatorial, procedeu-se a análise de Rasch, com o modelo de créditos parciais, com o uso do programa Winsteps, considerando a mesma estrutura fatorial usada com universitários. A comparação da dificuldade dos itens, theta, infit, outfit, erro padrão das estimativas de theta e da dificuldade dos itens e precisão das escalas foi realizada separadamente por cada fator. A ordem de apresentação das análises por fator, apresentada nas Tabelas de 2 a 8 , é feita mantendo a sequência dos fatores descrita 
no manual do teste, conforme apresentado na seção do Método deste artigo. A Tabela 2 apresenta a comparação dos parâmetros de Rasch entre universitários e alunos do EM, na dimensão Ciências Exatas.

Tabela 2- Medidas de ajuste e de erro padrão dos itens e das pessoas na dimensão Ciências Exatas

\begin{tabular}{|c|c|c|c|c|c|c|c|c|c|}
\hline \multirow[t]{2}{*}{ Dimensão } & \multicolumn{2}{|r|}{ Itens } & \multicolumn{7}{|c|}{ Pessoas } \\
\hline & & Dificuldade & Erro & Infit & Outfit & Theta & Erro & Infit & Outfit \\
\hline \multirow{2}{*}{$\begin{array}{l}\text { Ciências } \\
\text { Universitários }\end{array}$} & M & 0,04 & 0,04 & 1,02 & 1,04 & $-0,53$ & 0,32 & 1,06 & 1,03 \\
\hline & DP & 0,22 & 0,00 & 0,22 & 0,31 & 1,03 & 0,15 & 0,53 & 0,54 \\
\hline \multirow{2}{*}{$\begin{array}{l}\text { Ciências Exatas- } \\
\text { EM }\end{array}$} & M & 0,00 & 0,03 & 1,01 & 1,03 & $-0,64$ & 0,30 & 1,05 & 1,03 \\
\hline & DP & 0,22 & 0,00 & 0,20 & 0,31 & 0,82 & 0,13 & 0,52 & 0,52 \\
\hline
\end{tabular}

Foi possível verificar que todos os parâmetros analisados estiveram em níveis muito próximos, o que sugere que, para a dimensão Ciências Exatas, o padrão de resposta de universitários e alunos do EM é muito semelhante. A Tabela 3 apresenta a comparação da análise de Rasch para a dimensão Artes e Comunicação.

Tabela 3- Medidas de ajuste e de erro padrão dos itens e das pessoas na dimensão Artes e Comunicação

\begin{tabular}{|c|c|c|c|c|c|c|c|c|c|}
\hline \multirow[t]{2}{*}{ Dimensão } & \multicolumn{2}{|r|}{ Itens } & \multicolumn{7}{|c|}{ Pessoas } \\
\hline & & Dificuldade & Erro & Infit & Outfit & Theta & Erro & Infit & Outfit \\
\hline \multirow{2}{*}{$\begin{array}{l}\text { Artes/Comunicação- } \\
\text { Universitários }\end{array}$} & M & 0,00 & 0,03 & 1,01 & 1,04 & $-0,25$ & 0,27 & 1,05 & 1,04 \\
\hline & DP & 0,18 & 0,18 & 0,17 & 0,25 & 0,78 & 0,11 & 0,49 & 0,52 \\
\hline \multirow{2}{*}{$\begin{array}{l}\text { Artes/Comunicação- } \\
\text { do EM }\end{array}$} & M & 0,00 & 0,03 & 1,01 & 1,03 & $-0,40$ & 0,27 & 1,05 & 1,03 \\
\hline & DP & 0,17 & 0,00 & 0,19 & 0,28 & 0,74 & 0,11 & 0,46 & 0,47 \\
\hline
\end{tabular}

De modo semelhante ao que ocorreu com a dimensão Ciências Exatas, os indicadores analisados tiveram valores muito próximos nos dois grupos, para a dimensão Artes e Comunicação. Contudo, houve uma diferença na média de theta entre os grupos, que foi analisada por meio do teste $t$ de Student. Como resultado, a diferença entre os thetas dos dois grupos não foi estatisticamente significativa. $\mathrm{O}$ fato da média de theta ser menor no grupo de alunos do EM sugere que o interesse médio desse grupo por essa dimensão foi menor que a média de theta dos universitários. A Tabela 4 apresenta a análise de Rasch da dimensão Ciências Biológicas e da Saúde. 
Tabela 4- Medidas de ajuste e de erro padrão dos itens e das pessoas na dimensão Ciências Biológicas e da Saúde.

\begin{tabular}{|c|c|c|c|c|c|c|c|c|c|}
\hline \multirow[t]{2}{*}{ Dimensão } & \multicolumn{2}{|c|}{ Itens } & \multicolumn{7}{|c|}{ Pessoas } \\
\hline & & lade & Erro & Infit & Outfit & Theta & Erro & Infit & Outfit \\
\hline Ciências Biológicas e da & $\mathrm{M}$ & 0,00 & 0,04 & 1,01 & 1,05 & 0,11 & 0,38 & 1,03 & 1,04 \\
\hline Saúde - Universitários & DP & 0,46 & 0,00 & 0,12 & 0,16 & 1,07 & 0,14 & 0,57 & 0,71 \\
\hline Ciências Biológicas e da & $\mathrm{M}$ & 0,00 & 0,03 & 1,01 & 1,02 & $-0,20$ & 0,37 & 1,01 & 1,02 \\
\hline Saúde- Alunos do EM & DP & 0,27 & 0,00 & 0,11 & 0,13 & 1,03 & 0,15 & 0,55 & 0,61 \\
\hline
\end{tabular}

$\mathrm{Na}$ dimensão Ciências Biológicas e da Saúde, os indicadores de Rasch também foram muito semelhantes entre os dois grupos, com uma pequena exceção para a média de theta (0,11 para universitários e $-0,20$ para alunos do EM). Essa diferença, contudo, não foi estatisticamente significativa, quando analisada por meio do teste $t$ de Student. A Tabela 5 apresenta a análise da dimensão Ciências Agrárias/ambientais.

Tabela 5- Medidas de ajuste e de erro dos itens e das pessoas na dimensão Ciências agrárias/ ambientais.

\begin{tabular}{|c|c|c|c|c|c|c|c|c|c|}
\hline \multirow{2}{*}{ Dimensão } & \multicolumn{2}{|r|}{ Itens } & \multicolumn{7}{|c|}{ Pessoas } \\
\hline & & Dificuldade & Erro & Infit & Outfit & Theta & Erro & Infit & Outfit \\
\hline Ciênci & $\mathrm{M}$ & 0,00 & 0,04 & 1,01 & 1,04 & $-0,18$ & 0,30 & 1,04 & 1,04 \\
\hline Universitários & DP & 0,44 & 0,00 & 0,18 & 0,21 & 0,89 & 0,09 & 0,53 & 0,57 \\
\hline Ciências agrárias/ambie & M & 0,00 & 0,03 & 1,01 & 1,05 & $-0,20$ & 0,30 & 1,04 & 1,05 \\
\hline EM & DP & 0,37 & 0,00 & 0,22 & 0,28 & 0,91 & 0,09 & 0,55 & 0,60 \\
\hline
\end{tabular}

Do mesmo modo como já verificado nas outras dimensões, na presente as medidas dos itens e das pessoas estiveram em patamares semelhantes nos dois grupos. Na sequência, são apresentados na Tabela 6 os resultados da análise de Rasch da dimensão Atividades Burocráticas.

Tabela 6. Medidas de ajuste e de erro dos itens e das pessoas na dimensão Atividades Burocráticas.

\begin{tabular}{lllllllllll}
\hline Dimensão & \multicolumn{4}{c}{ Itens } & \multicolumn{1}{c}{ Pessoas } \\
& & Dificuldade & Erro & Infit & Outfit & Theta & Erro & Infit & Outfit \\
\hline Atividades Burocráticas- Universitários & $\mathrm{M}$ & 0,00 & 0,03 & 1,01 & 1,03 & $-0,12$ & 0,27 & 1,02 & 1,03 \\
& $\mathrm{DP}$ & 0,38 & 0,00 & 0,11 & 0,14 & 0,75 & 0,08 & 0,51 & 0,54 \\
Atividades Burocráticas- Alunos do EM & $\mathrm{M}$ & 0,00 & 0,03 & 1,01 & 1,03 & $-0,34$ & 0,27 & 1,04 & 1,03 \\
& $\mathrm{DP}$ & 0,24 & 0,00 & 0,19 & 0,26 & 0,67 & 0,08 & 0,51 & 0,51 \\
\hline
\end{tabular}

De modo análogo ao ocorrido nas demais dimensões da EAP, os parâmetros analisados dos itens e das pessoas nas Atividades Burocráticas foram semelhantes nos dois grupos. A diferença verificada em theta $(-0,12$ para universitários e -0,34 em alunos do EM) não foi estatisticamente significativa. A Tabela 7 apresenta a análise das Ciências Humanas e Sociais Aplicadas. 
Tabela 7 - Medidas de ajuste e de erro padrão dos itens e das pessoas na dimensão Ciências Humanas/Sociais aplicadas.

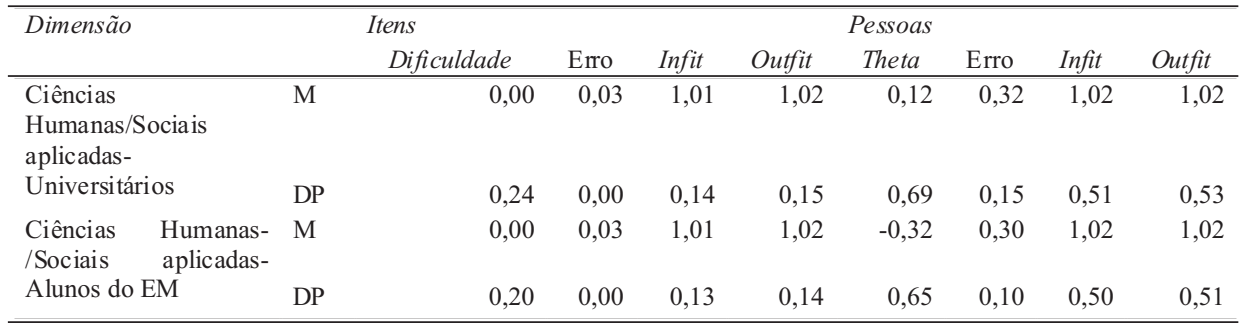

Na dimensão Ciências Humanas e Sociais Aplicadas obteve-se um resultado muito parecido, ao se comparar as informações de universitários e alunos do ensino médio. Assim como nas anteriores, nos quais houve pequenas diferenças na média de theta dos dois grupos, para a dimensão Ciências Humanas e Sociais Aplicadas essa diferença não foi estatisticamente significativa. Por fim, é apresentada a análise de Rasch da dimensão Entretenimento, na Tabela 8.

Tabela 8- Medidas de ajuste e de erro padrão dos itens e das pessoas na dimensão Entretenimento.

\begin{tabular}{|c|c|c|c|c|c|c|c|c|c|c|}
\hline \multirow{2}{*}{\multicolumn{2}{|c|}{ Dimensão }} & \multicolumn{2}{|r|}{ Itens } & \multicolumn{7}{|c|}{ Pessoas } \\
\hline & & & Dificuldade & Erro & Infit & Outfit & Theta & Erro & Infit & Outfit \\
\hline \multirow{2}{*}{\multicolumn{2}{|c|}{$\begin{array}{l}\text { Entretenimento- } \\
\text { Universitários }\end{array}$}} & M & 0,00 & 0,04 & 1,00 & 1,05 & $-0,22$ & 0,45 & 1,04 & 1,05 \\
\hline & & DP & 0,20 & 0,00 & 0,28 & 0,33 & 0,98 & 0,16 & 0,70 & 0,72 \\
\hline \multirow{2}{*}{$\begin{array}{l}\text { Entretenimento- } \\
\text { EM }\end{array}$} & Alunos do & M & 0,00 & 0,03 & 1,01 & 1,03 & $-0,32$ & 0,45 & 1,02 & 1,03 \\
\hline & & DP & 0,22 & 0,00 & 0,29 & 0,35 & 0,96 & 0,16 & 0,63 & 0,66 \\
\hline
\end{tabular}

A comparação dos parâmetros dos itens e das pessoas nos dois grupos evidenciou índices muito próximos, sugerindo que não houve diferenças nos padrões de resposta dos dois grupos para a dimensão Entretenimento. Em síntese, pode-se dizer que a análise das sete dimensões da EAP por Rasch sugere não haver diferença entre os padrões de resposta de universitários e alunos do Ensino Médio.

No que diz respeito ao infit e outfit dos itens e das pessoas, eles estiveram muito próximos de 1 em todas as dimensões para alunos do Ensino Médio, a média do erro padrão da dificuldade dos itens foi de 0,03 em todos os casos, enquanto o erro padrão de theta variou entre 0,27 e 0,45 . Quanto à média de theta nas dimensões, ainda considerando os dados dos alunos do EM, esse variou entre $-0,64$ e $-0,20$. O fato do theta assumir valores negativos indica que a média do traço latente (interesse) das pessoas encontra-se abaixo da dificuldade dos itens, que é situada em 0 pelo programa Winsteps. Em outras palavras, o conteúdo dos itens (sua dificuldade) sugere um nível de interesse mais elevado que o demonstrado por parte dos estudantes do EM, talvez indicando que os itens desta escala sejam muito específicos para pessoas com essa faixa etária, que possivelmente têm pouco contato ou informação sobre tais atividades. A análise que se seguiu 
foi da precisão das dimensões, separadamente para Universitários e alunos do ensino médio, conforme apresentado na Tabela 9. Presentemente foi considerada a precisão real dos itens e das pessoas, e não a precisão do modelo.

Tabela 9- Comparação da precisão dos itens e das pessoas pelo Alfa de Cronbach

\begin{tabular}{lrrrr}
\hline Dimensões & \multicolumn{2}{c}{ Universitários } & \multicolumn{2}{c}{ Alunos do EM } \\
& Itens & Pessoas & Itens & Pessoas \\
\hline Ciências Exatas & 0,97 & 0,88 & 0,98 & 0,80 \\
Artes e Comunicação & 0,97 & 0,86 & 0,97 & 0,81 \\
Ciências Biológicas e da Saúde & 0,99 & 0,86 & 0,98 & 0,82 \\
Ciências agrárias/ambientais & 0,99 & 0,88 & 0,99 & 0,85 \\
Atividades Burocráticas & 0,99 & 0,86 & 0,98 & 0,78 \\
Ciências Humanas/Sociais aplicadas & 0,98 & 0,79 & 0,98 & 0,71 \\
Entretenimento & 0,97 & 0,76 & 0,98 & 0,70 \\
\hline
\end{tabular}

É possível verificar que a precisão dos itens manteve um patamar muito elevado (todas acima de 0,97 tanto para universitários como para os Alunos do EM). A precisão dos itens, para alunos do EM, esteve entre 0,97 e 0,99 e das pessoas, entre 0,70 e 0,85 . No caso da precisão das pessoas, houve uma diminuição em todas as dimensões quando se tratou de alunos do EM, porém todas as dimensões apresentaram Alfa superior a 0,70 . Na sequência, os resultados serão discutidos à luz da literatura.

\section{DisCUSSÃo}

O objetivo deste estudo foi analisar a adequação da interpretação das dimensões da EAP em alunos do EM. Pesquisas desta natureza são especialmente relevantes ao se considerar que amostras diferentes poderão apresentar padrões de comportamento distintos quando analisados certos construtos, não sendo recomendado fazer inferências de que os testes podem ser igualmente úteis para amostras distintas, sem fundamentação empírica (AMERICAN EDUCATIONAL RESEARCH ASSOCIATION; AMERICAN PSYCHOLOGICAL ASSOCIATION; NATIONAL COUNCIL ON MEASUREMENT IN EDUCATION, 1999).

A importância dos estudos sobre os interesses vocacionais tem sido reafirmada por diversos autores, como Mattiazzi (1977), Bohoslavsky (1993), Leitão e Miguel (2004), Nunes et al. (2008b), entre outros. Os interesses possuem definições variadas, sendo que presentemente foram entendidos como padrões de atração ou evitação a atividades ocupacionais específicas. Para que teoria e pesquisa sobre os interesses possam ser conduzidos de forma satisfatória, é importante que haja instrumentos de medida psicometricamente adequados, para que se avance de maneira integrada na teoria e prática, indo ao encontro de lacunas já apontadas por alguns autores (NORONHA; AMBIEL, 2006; NORONHA et al., 2006, TEIXEIRA et al., 2007; SISTO; NORONHA; SANTOS, 2011).

Com vistas a verificar a adequação do EAP para a análise dos interesses em alunos do EM, foi realizada a análise fatorial da escala, tendo sido possível manter a estrutura fatorial original, com sete fatores, que explicaram 54\% da variância total. 
Esses fatores apresentaram precisão dos itens entre 0,97 e 0,99 , e das pessoas, entre 0,70 e 0,85 . Os valores podem ser considerados satisfatórios, segundo parâmetros da área (AERA; APA; NCME, 1999). Vale destacar, contudo, que a precisão das pessoas nos fatores Ciências Humanas e Sociais Aplicadas e Entretenimento foram as mais baixas, sugerindo a possibilidade de revisão desses fatores (por exemplo, inclusão de novos itens) para o uso com alunos do Ensino Médio.

Quanto à análise de Rasch conduzida com o EAP, foi possível verificar que os parâmetros infit, outfit e erro padrão das estimativas dos itens e das pessoas se mantiveram em patamares semelhantes em todas as dimensões e de acordo com o recomendado pela literatura (WRIGHT; STONE, 2004). Do mesmo modo, as médias de theta estiveram muito próximas em todas as dimensões, quando se comparou os dados de alunos do EM com universitários. Vale destacar que a média de theta esteve um pouco abaixo da dificuldade dos itens em todas as dimensões, sugerindo que os itens precisariam de pessoas com theta mais elevado para aderirem ao seu conteúdo. Em outras palavras, os itens possuíam, em média, conteúdo com adesão mais "difícil" que a média do nível de interesse demonstrado pelos alunos analisados.

Diferenças associadas à faixa etária do EAP já haviam sido investigadas por Sartori, Noronha e Nunes (2009), sendo que, embora tenham sido encontrados resultados que revelaram médias distintas, apenas a dimensão Artes e Comunicação apresentou diferença significativa. O presente estudo sugere que a estrutura fatorial originalmente proposta pelos autores da escala mantém-se, de modo geral, adequada para a aplicação com adolescentes, não parecendo ser necessária a interpretação dos resultados de interesses por meio de uma estrutura distinta. Os dados da análise de Rasch sugerem não haver diferenças nos padrões de resposta de alunos do ensino médio, quando comparados a universitários. Esse resultado coincide com as asserções de autores como Holland (1959), Lent, Brown e Hackett (1994) e Savickas (1995; 1999), no sentido de que não há diferenças na configuração dos interesses associada à idade. Desse modo, destaca-se que a análise das sete dimensões propostas no manual do EAP manteve-se adequada quando se considerou alunos do ensino médio e, portanto, o teste aparenta ser apropriado para uso com essa população.

Quanto às limitações do estudo, os alunos de EM representaram apenas dois estados brasileiros, sendo interessante a análise de dados oriundos de outras regiões do país. Outra consideração pertinente é que a precisão das pessoas nas dimensões Ciências Humanas e Sociais Aplicadas e Entretenimento esteve abaixo de 0,80 , tal como já destacado, o que pode tornar relevante a criação de novos itens para essas dimensões, de modo a tentar avaliar esses aspectos com maior precisão. De modo geral, esse estudo sugere a possibilidade de utilização do EAP com adolescentes, sendo recomendado, contudo, a criação de novas normas, buscando adequação às características dessa população. 


\section{REFERÊNCIAS}

AMERICAN EDUCATIONAL RESEARCH ASSOCIATION; AMERICAN PSYCHOLOGICAL ASSOCIATION; NATIONAL COUNCIL ON MEASUREMENT IN EDUCATION Standards for Educational and Psychological Testing. Washington, DC: [s.n.], 1999.

ANGELINI, A.L. O papel dos interesses na escolha da profissão. Psicologia Educacional 5. São Paulo, Boletim nº 185, 1957.

BAKER, F. B. The basics of Item Theory Response. Washington, DC: ERICClearinghouse on Assessment and Evaluation, 2001.

BOHOSLAVSKY, R. Orientação vocacional: a estratégia clínica. São Paulo: Martins Fontes, 1993.

BORDIN, E. G. A theory of interests as dynamic phenomena. Educational and Psychological Measurement, [S.1.], v. 3, p. 49-66, 1943.

CARTER, H. D. The development of vocational attitudes. Journal of Consulting Psychology, [S.1.], v. 4, n. 5, p. 185-191, Sept. 1940.

ELLIOT, T. R. et al. Deconstructing therapy outcome measurement with Rasch analysis of a measure of general clinical distress: the Symptom Checklist-90 Revised. Psychological Assessment, v. 18, n. 4, p. 359-372, 2006.

EMBRETSON, S.; REISE, S. Item Response Theory for Psychologists. Mahwah, New Jersey: Lawrence Erlbaum Associates, 2000.

FRYER, D. H. The measurement of interests. New York: Holt, 1931.

HOLLAND, J. L. A theory of vocational choice. Journal of Counseling Psychology, v. 6, n. p. 35-45, 1959.

HOLLAND, J. L. Explorations of a theory of vocational choice and achievement: a four-year prediction study. Psychological Reports, v. 12, p. 547-594, 1963.

HOLlAnD, J. L.; FRITZSCHE, B. A.; POWELL, A. B. Sds- Self- Directed Search. Los Angeles, California: PAR- Psychological Assessment Resources, 1994.

LEITÃO, L. M.; MIGUEL, J. P. Avaliação dos interesses. In: Leitão, L. M. (Ed.), Avaliação psicológica em orientação escolar e profissional. Coimbra: Quarteto, 2004. p. 179-262. 
LENT, R.; BROWN, S. D.; HACKETT, G. Toward a unifying social cognitive theory of career and academic interest, choice and performance. Journal of Vocational Behavior, v. 45, n. 1, p. 79-122, 1994.

LINACRE, J. M. Kr-20 / Cronbach alpha or Rasch reliability: which tells the "truth"? Rasch Measurement Transactions, v. 11, n. 3 p. 580-581, 1997.

MATTIAZI, B. A natureza dos interesses e a orientação vocacional. Petrópolis, RJ: Vozes, 1977.

NORONHA, A. P.; SISTO, F.; SANTOS, A. A. A. Escala de aconselhamento profissional- EAP- manual técnico. Itatiba-SP: Vetor, 2007.

NORONHA, A. P. P.; AMBIEL, R. A. M. Orientação profissional e vocacional: análise da produção científica. PsicoUsf, v. 11, n. 1, p. 75-84, 2006.

NORONHA, A. P. P. et al. Análise de teses e dissertações em orientação profissional. Revista da $A B O P$, v. 7, n. 2, p. 1-10, 2006.

NUNES, C. H. S. S. et al. Teoria de Resposta ao Item para otimização de escalas tipo Likert: um exemplo de aplicação. Revista Iberoamerica de Diagnóstico y Evaluación Psicológica, v. 25, n. 1, p. 51-79, 2008 a.

NUNES, M. F. O. et al. Interesses profissionais: perspectivas teóricas e instrumentos de avaliação. Avaliação Psicológica, v. 7, n. 3, p. 403-414, 2008 b.

PASQUALI, L. Psicometria: teoria dos testes na Psicologia e na Educação. Petrópolis, RJ: Vozes, 2003.

PASQUALI, L.; PRIMI, R. Fundamentos da Teoria da Resposta ao Item - TRI. Avaliação Psicológica, v. 2, n. 2, p. 99-110, 2003.

RASCH, G. Probablistic Models for Some Intelligence and Attainment Tests. Chicago: University of Chicago, 1960.

SARTORI, F. A.; NORONHA, A. P. P.; NUNES, M. F. O. Comparações entre Eap e Sds: interesses profissionais em alunos de ensino médio. Boletim de Psicologia, v. LIX, n. p. 17-29, 2009.

SAVICKAS, M. L. Examining the personal meaning of inventoried interests during career counseling. Journal of Career Assessment, v. 3, n. 2, p. 188-201, 1995. 
SAVICKAS, M. L. The Psychology of Interests. In: SAVICKAS, M. L.; SPOKANE, A. R. (Ed.). Vocational interests: meaning, measurement and counseling use. Palo Alto, CA: Davies-Black, 1999. p. 19-56.

SISTO, F. F.; NORONHA, A. P. P.; SANTOS, A. A. A. Escala de Auto-Eficácia para Escolha Profissional: avaliação preliminar das propriedades psicométricas. Psicología para América Latina, v. 21, n. 2, p. 38-43, 2011.

STRONG, E. K. Jr. Vocational interests of men and women. Stanford, CA: Stanford University, 1943.

TEIXEIRA, M. A. P. et al. Produção científica em orientação profissional: uma análise da Revista Brasileira de Orientação Profissional. Revista Brasileira de Orientação Profissional, v. 8, n. 2, p. 25-40, 2007.

TYLER, L. E. The relationship of interests to abilities and regulation among firstgrade. Psychological Measurement, [S.1.], v. 3, p. 49-66, 1951.

WRIGHT, B. D.; STONE, M. H. Making Measures. Chicago, IL: Phaneron, 2004.

Recebido em: 26 de janeiro de 2010 Aceito em: 21 de março de 2012 